\title{
Ethnic discrimination prevalence and associations with health outcomes: data from a nationally representative cross-sectional survey of secondary school students in New Zealand
}

\author{
Sue Crengle ${ }^{1 *}$, Elizabeth Robinson ${ }^{2}$, Shanthi Ameratunga ${ }^{2}$, Terryann Clark $^{3}$ and Deborah Raphae ${ }^{3}$
}

\begin{abstract}
Background: Reported ethnic discrimination is higher among indigenous and minority adult populations. There is a paucity of nationally representative prevalence studies of ethnic discrimination among adolescents. Experiencing ethnic discrimination has been associated with a range of adverse health outcomes. NZ has a diverse ethnic population. There are health inequalities among young people from Māori and Pacific ethnic groups.

Methods: 9107 randomly selected secondary school students participated in a nationally representative crosssectional health and wellbeing survey conducted in 2007. The prevalence of ethnic discrimination by health professionals, by police, and ethnicity-related bullying were analysed. Logistic regression was used to examine the associations between ethnic discrimination and six health/wellbeing outcomes: self-rated health status, depressive symptoms in the last 12 months, cigarette smoking, binge alcohol use, feeling safe in ones neighbourhood, and self-rated school achievement.

Results: There were significant ethnic differences in the prevalences of ethnic discrimination. Students who experienced ethnic discrimination were less likely to report excellent/very good/good self-rated general health (OR

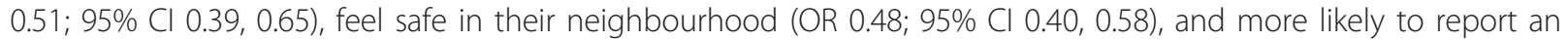
episode of binge drinking in the previous 4 weeks $(\mathrm{OR} \mathrm{1.77;95 \%} \mathrm{Cl} \mathrm{1.45,2.17).} \mathrm{For} \mathrm{all} \mathrm{these} \mathrm{outcomes} \mathrm{the} \mathrm{odds}$ ratios for the group who were 'unsure' if they had experienced ethnic discrimination were similar to those of the 'yes' group.

Ethnicity stratified associations between ethnic discrimination and the depression, cigarette smoking, and selfrated school achievement are reported. Within each ethnic group participants reporting ethnic discrimination were more likely to have adverse outcomes for these three variables. For all three outcomes the direction and size of the association between experience of ethnic discrimination and the outcome were similar across all ethnic groups.
\end{abstract}

Conclusions: Ethnic discrimination is more commonly reported by Indigenous and minority group students. Both experiencing and being 'unsure' about experiencing ethnic discrimination are associated with a range of adverse health/wellbeing outcomes. Our findings highlight the progress yet to be made to ensure that rights to be free from ethnic discrimination are met for young people living in New Zealand.

\footnotetext{
* Correspondence: s.crengle@auckland.ac.nz

${ }^{1}$ Te Kupenga Hauora Māori, School of Population Health, Faculty of Medical and Health Sciences, University of Auckland, Private Bag 92019, Wellesley St, Auckland, New Zealand

Full list of author information is available at the end of the article
} 


\section{Background}

Racism, the belief that some population groups ('races' or ethnic groups) are superior to others, ensures that power and privilege within society is conferred on the group(s) considered superior $[1,2]$. Ethnic discrimination, the behavioural manifestation of racism, is defined as "unfair, differential treatment on the basis of race or ethnicity" [3]. Discriminatory acts between individuals constitute interpersonal discrimination while discriminatory policies or practices embedded in organisational structures constitute institutional discrimination [4-6].

The majority of ethnic discrimination research has been undertaken in adult populations. Among adult populations in the US, UK, Australia and New Zealand, reported ethnic discrimination is higher among indigenous and minority populations [4,7-10]. There is a paucity of nationally representative prevalence studies of ethnic discrimination among adolescents. Experiencing ethnic discrimination has been associated with a range of adverse physiological, physical, psychological, and behavioural outcomes among adult and adolescent populations including: poor self-reported overall health status [4,11-14]; depression, somatic complaints, anxiety and psychosis $[3,7,12-21]$; and risk-taking behaviours such as smoking, increased alcohol consumption, and use of other psychoactive substances [14,20-23]. Experiencing discrimination has also been shown to be associated with ethnic disparities in access to, and quality of care [14]. In NZ, self-reported discrimination has been found to account for some of the observed health inequalities between Māori and NZ European adults [24].

New Zealand has a diverse population. The 2006 population census enumerated 606207 people aged 1019 years. Māori (the indigenous people of New Zealand) accounted for $20.7 \%$ of this age group, with $9.8 \%$ identifying Pacific ethnic groups, 9.9\% Asian ethnic groups, $62.4 \%$ European and $9.0 \%$ as other ethnic group. Note that percentages add up to more than 100 as people who identified with more than one ethnic group have been counted in all ethnic groups they identified.

New Zealand youth health statistics leave much to be desired with rates of suicide, death from motor vehicle crashes, and alcohol and other substance use among some of the highest in the Western world [25]. Furthermore, there are significant ethnic differences in health and wellbeing outcomes. Compared to non-Māori, Māori youth aged 15-24 years have significantly higher mortality rates from accidents and suicide [26] and significantly higher rates of hospitalisation for injury/poisoning and mental/behavioural disorders [27]. Greater proportions of Pacific and Māori secondary school students reported smoking cigarettes at least weekly, using marijuana at least weekly, and having made a suicide attempt in the previous 12 months [28,29].

The purpose of this paper is to contribute to the literature about ethnic discrimination among adolescents by reporting the findings from a nationally representative survey of young people attending secondary school in New Zealand. We describe the self-reported prevalence of experience of ethnic discrimination and the associations between ethnic discrimination and health and wellbeing outcomes.

\section{Methods}

Youth'07 The Second National Health and Wellbeing Survey of New Zealand Secondary School Students (Youth'07) is a nationally representative cross-sectional survey of the health and wellbeing of Year 9 to $13 \mathrm{sec}-$ ondary school students in New Zealand (NZ) conducted in 2007. The study design, described in detail elsewhere [30], is summarised here. A two-stage clustered sampling design was used. One hundred and fifteen randomly selected schools were invited to participate and, in each school, students were randomly selected and invited to participate. The response rate for schools was $84 \%$ and for students was $74 \%$. In total, 9107 students completed the survey, accounting for $3.4 \%$ of the total number of students enrolled in secondary schools in 2007. All data were collected during school days, in designated rooms in each school using Multimedia Computer Assisted Self Interview (M-CASI). Each student received a login code to access the anonymous 622 item multimedia survey which was self-administered using hand-held internet tablets [31]. Written consent to participate was obtained from each participating school. Written information about the study was given to the selected students and their parents. On the day of the survey each student provided consent to participate. The University of Auckland Human Subjects Ethics Committee approved the study.

Self-reported ethnicity data was collected according to the Statistics New Zealand standard for the electronic collection of ethnicity data [32]. Students were able to choose multiple ethnic groups. Where this was the case ethnicity data were categorised into a single ethnic group using a hierarchical prioritisation method that prioritised ethnicity in the following order: Māori, Pacific, Asian, Other, or NZ European [33]. Māori are the indigenous people of NZ. Pacific ethnic groups include Samoan, Tongan, Niuean, Cook Island Māori and other Pacific Islands. The Other ethnic group compromises all ethnic groups that are not included in the Māori, Pacific, Asian and NZ European categories. The Asian ethnic group includes participants from Asia and the Indian sub-continent. The 'other' ethnic group is 
extremely diverse and includes participants from Middle East, Africa, South America, North America, and counties in Europe.

Three socio-economic variables were included in the analyses: the New Zealand Index of Deprivation 2006 (NZDep2006) decile [34], food security, and housing mobility. NZDep2006 deciles were categorised into three deprivation groups: low (Index deciles 1-3), moderate (4-7), and high deprivation (8-10). Food security was assessed by asking 'Do your parents, or the people who act as your parents, ever worry about not having enough money to buy food?' and responses were categorised into 'never', 'occasionally/sometimes', and 'often/all the time'. Housing mobility was assessed by asking 'In the past year how many times have you moved homes?' and responses were dichotomised into 'none or one time' and 'two or more times'. Age was categorised into five discrete levels: $\leq 13$ years, $14,15,16$ and $\geq 17$ years.

Students' experiences of ethnic discrimination in three settings were examined: unfair treatment by the police because of ethnicity, unfair treatment by health professionals because of ethnicity, and bullying because of ethnicity. Response options for the unfair treatment variables were 'no', 'yes', and 'unsure' and the time period was 'ever' experienced these types of discrimination. Participants who reported they had been bullied were asked to indicate the reason(s) for bullying from a dropdown list that included 'I was bullied because of my ethnic group or culture'. The timeframe for 'ethnicityrelated bullying' is 'in the current school year'. A summary variable, 'any ethnic discrimination', was developed to assess the overall experience of ethnic discrimination.

Six measures were used to assess the impact of selfreported discrimination on health and wellbeing: selfrated health status, depressive symptoms in the last 12 months, cigarette smoking at least weekly, binge alcohol use in the previous 4 weeks, feeling safe in ones neighbourhood, and self-rated achievement at school. Selfrated health was dichotomised into 'excellent/very good/ good' and 'fair/poor'. The Reynolds Adolescent Depression Scale (RADS) which measures depressive symptomatology in young people aged 12 to 18 years [35], has been validated for use in the NZ population both in its original [36] and in the short form [37]. Assessment of the short form version (RADS-SF) found that a cut off of 28 for depressive symptoms gave best agreement with the prevalence of depressive symptoms determined by the long form of the RADS. Accordingly we have used a RADS-SF score of $\geq 28$ to identify students with significant depressive symptoms [37]. Cigarette smoking was categorised into students who smoked at least weekly and those who had never smoked or smoked less than once per week. Binge drinking was defined as having five or more alcoholic drinks in one session within a 4 hour period. The binge drinking variable was categorised as students who did not drink binge drink in the previous 4 weeks and those who had at least one episode of binge drinking in the previous 4 weeks. Students were asked if they felt safe in their neighbourhood and data were dichotomised into 'feeling safe all/most of the time' and 'sometimes/mostly not/not feeling safe'. Self-rated achievement at school was assessed by asking students how well they did at school with dichotomisation of responses into 'near the top/above the middle/about the middle' and 'below the middle/near the bottom'.

Only participants with data on discrimination variables have been included in the analyses. No imputation has been done for missing variables. Data was analysed using Statistical Analysis Software (Version 9.2; SAS Institute, North Carolina, USA). All analyses were adjusted for design effects (clustering and unequal probability of selection). Descriptive data analyses (frequency distributions, 95\% confidence intervals, Chi-square analyses) were stratified by ethnicity and by discrimination status. Logistic regression modelling was employed to analyse the associations between ethnicity and ethnic discrimination variables and the associations between ethnic discrimination and health/wellbeing outcome variables while adjusting for confounding variables. New Zealand European students were used as the reference group in all logistic regression analyses.

\section{Results}

Ethnicity data were available for 9080 of the 9107 participating students. NZ European was the most frequently reported ethnic group (53\%) followed by Māori (19\%), Asian (13\%), Pacific (10\%) and other ethnic groups (6\%). The median age was 14 years with approximately $99 \%$ of students aged between 13 and 18 years and about $1 \%$ aged less than 13 years or over 18 years of age (data not shown). There were significant ethnic differences in age distribution with lower proportions of Māori, Pacific and Other ethnic groups in the older ages. Significant ethnic differences in socioeconomic variables were observed with higher proportions of Pacific and Māori students living in high deprivation areas, reporting food insecurity, and reporting housing mobility. (Table 1)

Ethnicity-related bullying was most commonly reported by Asian participants. Māori, Pacific and Other ethnic groups were also significantly more likely to report this experience than the NZ European ethnic group. Pacific, Māori, Asian and Other ethnic groups were significantly more likely to report experiencing ethnic discrimination by the police than NZ European participants. Māori, Pacific and Asian participants were significantly more likely to report being unsure if they had experienced ethnic discrimination by the police than NZ European participants. Pacific, Asian, Māori 
Table 1 Sociodemographic data by ethnicity from Youth'07-national survey of secondary school student's health and wellbeing, New Zealand 2007

\begin{tabular}{|c|c|c|c|c|c|c|c|c|c|c|c|c|}
\hline & \multicolumn{2}{|r|}{ Māori } & \multicolumn{2}{|r|}{ Pacific } & \multicolumn{2}{|r|}{ Asian } & \multicolumn{2}{|r|}{ Other } & \multicolumn{2}{|c|}{ NZ European } & \multicolumn{2}{|r|}{ Total } \\
\hline & $\mathrm{n}$ & $\%$ & $\mathrm{n}$ & $\%$ & $\mathrm{n}$ & $\%$ & $\mathrm{n}$ & $\%$ & $\mathrm{n}$ & $\%$ & n & $\%$ \\
\hline & & $95 \% \mathrm{Cl}$ & & $95 \% \mathrm{Cl}$ & & $95 \% \mathrm{Cl}$ & & $95 \% \mathrm{Cl}$ & & $95 \% \mathrm{Cl}$ & & $95 \% \mathrm{Cl}$ \\
\hline \multirow[t]{3}{*}{ Total } & 1702 & & 924 & & 1126 & & 531 & & 479 & & 9080 & \\
\hline & & 18.7 & & 10.2 & & 12.5 & & 5.9 & 7 & 52.6 & & \\
\hline & & $15.6-21.9$ & & $6.4-14.1$ & & $7.6-17.4$ & & $5.0-6.8$ & & $47.6-57.7$ & & \\
\hline \multicolumn{13}{|l|}{ Sex } \\
\hline \multirow[t]{2}{*}{ Male } & 882 & 52.0 & 497 & 53.8 & 619 & 55.1 & 273 & 51.3 & 263 & 55.0 & 4902 & 54.1 \\
\hline & & $45.6-58.4$ & & $43.7-63.9$ & & $42.8-67.4$ & & $43.7-58.9$ & 1 & $47.4-62.5$ & & $47.5-60.7$ \\
\hline \multirow[t]{2}{*}{ Female } & 820 & 48.0 & 427 & 46.2 & 507 & 44.9 & 258 & 48.7 & 216 & 45.0 & 4178 & 45.9 \\
\hline & & $41.6-54.4$ & & $36.1-56.3$ & & $32.6-57.2$ & & $41.1-56.3$ & 6 & $37.5-52.6$ & & $39.3-52.5$ \\
\hline \multicolumn{13}{|l|}{ Age (years) } \\
\hline \multirow[t]{2}{*}{$\leq 13$} & 414 & 24.1 & 213 & 23.0 & 163 & 14.5 & 107 & 20.1 & 959 & 19.8 & 1856 & 20.3 \\
\hline & & $21.3-26.8$ & & $20.6-25.5$ & & $10.5-18.4$ & & $16.6-23.5$ & & $17.8-21.9$ & & $18.5-22.1$ \\
\hline \multirow[t]{2}{*}{14} & 434 & 25.6 & 201 & 21.6 & 211 & 18.7 & 109 & 20.3 & 113 & 23.7 & 2094 & 23.0 \\
\hline & & $23.2-27.9$ & & $19.1-24.2$ & & $16.8-20.7$ & & $16.0-24.6$ & 9 & $22.2-25.2$ & & $21.7-24.3$ \\
\hline \multirow[t]{2}{*}{15} & 387 & 22.8 & 196 & 21.3 & 221 & 19.5 & 133 & 25.2 & 103 & 21.6 & 1968 & 21.7 \\
\hline & & $20.9-24.7$ & & $18.5-24.1$ & & $17.8-21.2$ & & $21.1-29.3$ & 1 & $20.4-22.8$ & & $20.9-22.6$ \\
\hline \multirow[t]{2}{*}{16} & 268 & 15.8 & 182 & 19.7 & 252 & 22.4 & 107 & 20.2 & 932 & 19.5 & 1741 & 19.2 \\
\hline & & $14.2-17.5$ & & $17.1-22.4$ & & $19.6-25.3$ & & $17.2-23.2$ & & $18.1-20.8$ & & $18.1-20.3$ \\
\hline \multirow[t]{2}{*}{$\geq 17$} & 199 & 11.7 & 132 & 14.3 & 279 & 24.9 & 75 & 14.2 & 736 & 15.4 & 1421 & 15.7 \\
\hline & & $10.1-13.3$ & & $11.9-16.7$ & & $21.8-27.9$ & & $10.3-18.1$ & & $13.9-16.9$ & & $14.3-17.1$ \\
\hline \multicolumn{13}{|l|}{ NZDep2006 } \\
\hline \multirow[t]{2}{*}{ Low deprivation } & 334 & 20.3 & 70 & 7.8 & 424 & 38.6 & 219 & 42.4 & 216 & 46.4 & 3215 & 36.4 \\
\hline & & $16.3-24.2$ & & $4.6-11.0$ & & $29.6-47.5$ & & $33.3-51.5$ & 8 & $41.6-51.3$ & & $31.1-41.7$ \\
\hline \multirow[t]{2}{*}{ Medium deprivation } & 577 & 34.9 & 221 & 24.8 & 481 & 43.8 & 190 & 36.9 & 192 & 41.0 & 3395 & 38.3 \\
\hline & & $30.9-38.9$ & & $18.2-31.4$ & & $39.8-47.8$ & & $31.6-42.2$ & 6 & $37.7-44.4$ & & $35.5-41.2$ \\
\hline \multirow[t]{2}{*}{ High deprivation } & 743 & 44.9 & 601 & 67.4 & 194 & 17.6 & 107 & 20.7 & 594 & 12.5 & 2239 & 25.3 \\
\hline & & $38.3-51.4$ & & $58.4-76.4$ & & $9.4-25.9$ & & $13.9-27.5$ & & $10.2-14.9$ & & $19.8-30.7$ \\
\hline \multicolumn{13}{|c|}{$\begin{array}{l}\text { Parents worry about having } \\
\text { enough money to buy food }\end{array}$} \\
\hline \multirow[t]{2}{*}{ Never } & 869 & 54.8 & 362 & 43.5 & 740 & 71.2 & 362 & 43.5 & 311 & 70.1 & 5415 & 64.4 \\
\hline & & $52.3-57.4$ & & $38.6-48.4$ & & $66.6-75.7$ & & $38.6-48.4$ & 8 & $67.7-72.6$ & & $61.5-67.4$ \\
\hline Occasionally/sometimes & 553 & 34.7 & 336 & 40.4 & 233 & 22.2 & 336 & 40.4 & 109 & 24.3 & 2352 & 27.8 \\
\hline & & $32.2-37.2$ & & $36.0-44.9$ & & $19.0-25.4$ & & $36.0-44.9$ & 4 & $22.4-26.3$ & & $25.6-29.9$ \\
\hline Often/all the time & 165 & 10.5 & 134 & 16.1 & 70 & 6.6 & 134 & 16.1 & 251 & 5.6 & 659 & 7.8 \\
\hline & & $8.6-12.4$ & & $13.8-18.4$ & & $4.6-8.6$ & & $13.8-18.4$ & & $4.8-6.4$ & & $6.7-8.8$ \\
\hline $\begin{array}{l}\text { Moved home } 2 \text { or more } \\
\text { last } 12 \text { months }\end{array}$ & & & & & & & & & & & & \\
\hline Yes & 311 & 18.7 & 187 & 20.7 & 177 & 16.0 & 70 & 13.5 & 393 & 8.3 & 1138 & 12.8 \\
\hline & & $16.9-20.4$ & & $16.2-25.2$ & & $13.5-18.4$ & & $10.7-16.4$ & & $7.4-9.2$ & & $11.7-13.9$ \\
\hline No & 1362 & 81.3 & 718 & 79.3 & 933 & 84.0 & 451 & 86.5 & 433 & 91.7 & 7794 & 87.2 \\
\hline & & $79.6-83.1$ & & $74.8-83.8$ & & $81.6-86.5$ & & $83.6-89.3$ & 0 & $90.8-92.6$ & & $86.1-88.3$ \\
\hline
\end{tabular}

and Other ethnic groups were significantly more likely to report ethnic discrimination and being unsure about experiencing ethnic discrimination by health professionals than NZ European participants. Experiencing and being unsure about experiencing discrimination in one or more of the three dimensions ('any ethnic discrimination') were significantly more common among
Asian, Pacific, Māori and Other ethnic groups than NZ European. (Table 2)

In unadjusted bivariate analyses, self-reported experience of ethnic discrimination in each of the three dimensions and 'any ethnic discrimination' were related to adverse outcomes for all of the health and well-being variables under investigation. (Table 3). 
Table 2 Ethnic discrimination, crude prevalences by ethnicity and adjusted comparisons between ethnic groups, Youth'07

\begin{tabular}{|c|c|c|c|c|c|c|c|c|}
\hline & & & Maori & Pacific & Asian & Other & $\begin{array}{c}\mathrm{NZ} \\
\text { European }\end{array}$ & Total \\
\hline & & $n$ & 52 & 31 & 95 & 19 & 83 & 280 \\
\hline \multirow[t]{7}{*}{ Had experienced ethnicity-related bullying $N=8821$} & & $\%$ & 3.1 & 3.5 & 8.5 & 3.7 & 1.8 & 3.2 \\
\hline & Yes & $95 \% \mathrm{Cl}$ & $2.3-4.0$ & $2.2-4.8$ & $6.8-10.2$ & $1.7-5.8$ & $1.3-2.3$ & $2.7-3.7$ \\
\hline & & OR & 1.61 & 1.61 & 5.36 & 2.28 & & \\
\hline & & $95 \% \mathrm{Cl}$ & $1.01-2.56$ & $0.99-2.62$ & $3.75-7.67$ & $1.27-4.12$ & 1 & \\
\hline & & $\mathrm{n}$ & 96 & 58 & 57 & 20 & 99 & 330 \\
\hline & & $\%$ & 6.5 & 7.5 & 5.4 & 4.2 & 2.2 & 4.0 \\
\hline & Yes & $95 \% \mathrm{Cl}$ & $5.1-7.8$ & $5.0-10.1$ & $3.6-7.2$ & $2.5-5.9$ & $1.7-2.7$ & $3.4-4.6$ \\
\hline \multirow{10}{*}{$\begin{array}{l}\text { Treated unfairly by the police because of ethnicity } N \\
=8284\end{array}$} & & OR & 2.78 & 3.10 & 2.70 & 2.03 & & \\
\hline & & $95 \% \mathrm{Cl}$ & $2.03-3.80$ & $2.02-4.73$ & $1.75-4.16$ & $1.25-3.32$ & 1 & \\
\hline & & $n$ & 128 & 69 & 63 & 16 & 128 & 404 \\
\hline & & $\%$ & 8.6 & 9.0 & 6.0 & 3.2 & 2.8 & 4.9 \\
\hline & Unsure & $95 \% \mathrm{Cl}$ & $7.1-10.1$ & $7.1-10.9$ & $4.6-7.4$ & $2.0-4.5$ & $2.3-3.4$ & $4.2-5.5$ \\
\hline & & OR & 3.10 & 2.77 & 2.27 & 1.22 & & \\
\hline & & $95 \% \mathrm{Cl}$ & $2.30-4.17$ & $1.86-4.14$ & $1.63-3.17$ & $0.78-1.91$ & 1 & \\
\hline & & $n$ & 84 & 74 & 58 & 19 & 95 & 330 \\
\hline & & $\%$ & 5.0 & 8.6 & 5.3 & 3.7 & 2.0 & 3.8 \\
\hline & Yes & $95 \% \mathrm{Cl}$ & $4.1-6.0$ & $6.7-10.4$ & $4.0-6.5$ & $1.9-5.6$ & $1.5-2.6$ & $3.2-4.3$ \\
\hline \multirow{11}{*}{$\begin{array}{l}\text { Treated unfairly by health professional because of } \\
\text { your ethnicity } N=8785\end{array}$} & & OR & 2.41 & 3.82 & 3.17 & 2.03 & & \\
\hline & & $95 \% \mathrm{Cl}$ & $1.76-3.31$ & $2.63-5.57$ & $2.03-4.95$ & $1.09-3.76$ & 1 & \\
\hline & & $n$ & 248 & 117 & 230 & 73 & 386 & 1054 \\
\hline & & $\%$ & 15.0 & 13.5 & 20.9 & 14.4 & 8.3 & 12.0 \\
\hline & Unsure & $95 \% \mathrm{Cl}$ & $13.4-16.6$ & $10.9-16.1$ & $17.9-23.8$ & $10.9-17.9$ & $7.3-9.2$ & $11.0-13.0$ \\
\hline & & OR & 1.57 & 1.38 & 3.27 & 1.79 & & \\
\hline & & $95 \% \mathrm{Cl}$ & $1.32-1.86$ & $1.07-1.78$ & $2.76-3.89$ & $1.30-2.45$ & 1 & \\
\hline & & $n$ & 197 & 139 & 180 & 52 & 238 & 806 \\
\hline & & $\%$ & 12.9 & 17.4 & 16.7 & 10.8 & 5.4 & 9.7 \\
\hline & Yes & $95 \% \mathrm{Cl}$ & $11.0-14.8$ & $14.2-20.7$ & $14.0-19.4$ & $7.8-13.7$ & $4.5-6.2$ & $8.6-10.7$ \\
\hline & & OR & 2.49 & 3.18 & 4.54 & 2.48 & & \\
\hline \multirow[t]{6}{*}{ Any ethnic discrimination $N=8345$} & & $95 \% \mathrm{Cl}$ & $1.96-3.16$ & $2.34-4.32$ & $3.49-5.91$ & $1.82-3.38$ & 1 & \\
\hline & & $n$ & 290 & 136 & 220 & 79 & 431 & 1156 \\
\hline & & $\%$ & 19.1 & 17.0 & 20.6 & 16.3 & 9.6 & 13.9 \\
\hline & Unsure & $95 \% \mathrm{Cl}$ & $16.7-21.5$ & $14.2-19.8$ & $17.5-23.6$ & $12.5-20.0$ & $8.6-10.7$ & $12.7-15.1$ \\
\hline & & OR & 1.92 & 1.61 & 3.09 & 1.88 & & \\
\hline & & $95 \% \mathrm{Cl}$ & $1.58-2.33$ & $1.24-2.07$ & $2.60-3.69$ & $1.40-2.51$ & 1 & \\
\hline
\end{tabular}

Odds ratios compare the odds of reporting ethnic discrimination for each ethnic group using NZ European as the reference group and are adjusted for age, sex, NZDep2006, food security, housing mobility

Logistic regression analysis was used to explore the relationship between experiencing 'any ethnic discrimination' and the health and wellbeing outcomes after adjusting for age, sex, ethnicity and the three socioeconomic variables. Testing for interaction between ethnicity and 'any ethnic discrimination' was undertaken for all outcome variables. As there was no significant interaction for the general health, binge drinking and feeling safe in the neighbourhood variables the results of logistic regressions are reported for the total sample.
Compared with participants who did not experience ethnic discrimination, those who reported 'yes' or 'unsure' were significantly less likely to report excellent/very good/good self-rated general health, less likely to feel safe in their neighbourhood, and more likely to have had an episode of binge drinking in the previous four weeks. (Table 4).

Significant interactions between ethnicity and 'any ethnic discrimination' were observed for the depression ( $p$ $=0.009)$, smoking cigarettes $(p=0.04)$, and school 
Table 3 Health and wellbeing outcomes by ethnic discrimination, Youth'07

\begin{tabular}{|c|c|c|c|c|c|c|c|c|}
\hline & & & $\begin{array}{l}\text { General } \\
\text { excellent/ } \\
\text { very good/ } \\
\text { good }\end{array}$ & $\begin{array}{c}\text { RADS } \\
\text { significant } \\
\text { depression } \\
\text { symptoms }\end{array}$ & $\begin{array}{l}\text { Smokes } \\
\text { cigarettes } \\
\text { at least } \\
\text { weekly }\end{array}$ & $\begin{array}{c}\text { Binge } \\
\text { drinking } \\
\text { in last } 4 \\
\text { weeks }\end{array}$ & $\begin{array}{l}\text { Feels safe in } \\
\text { neighbourhood } \\
\text { all/most of } \\
\text { time }\end{array}$ & $\begin{array}{c}\text { Self-rated } \\
\text { achievement in } \\
\text { school } \\
\text { near top/above or } \\
\text { about the middle }\end{array}$ \\
\hline \multirow{6}{*}{$\begin{array}{l}\text { Had experienced ethnicity- } \\
\text { related bullying }\end{array}$} & Yes & $n$ & 241 & 64 & 26 & 78 & 168 & 244 \\
\hline & & $\%$ & 86.5 & 23.6 & 9.7 & 30.0 & 65.3 & 87.1 \\
\hline & & $95 \% \mathrm{Cl}$ & 82.3 - 90.7 & $17.7-29.5$ & $5.8-13.5$ & $23.8-36.3$ & $58.7-71.9$ & $83.4-90.8$ \\
\hline & No & $n$ & 7832 & 881 & 612 & 2738 & 6511 & 7807 \\
\hline & & $\%$ & 92.1 & 10.5 & 7.7 & 34.7 & 81.7 & 91.5 \\
\hline & & $95 \% \mathrm{Cl}$ & $91.3-92.9$ & $9.6-11.4$ & $6.8-8.5$ & $32.1-37.3$ & $80.0-83.3$ & $90.7-92.3$ \\
\hline \multirow{9}{*}{$\begin{array}{l}\text { Treated unfairly by the police } \\
\text { because of ethnicity }\end{array}$} & Unsure & $n$ & 345 & 97 & 64 & 168 & 255 & 343 \\
\hline & & $\%$ & 85.2 & 23.9 & 17.5 & 47.2 & 64.9 & 84.8 \\
\hline & & $95 \% \mathrm{Cl}$ & $81.7-88.8$ & $19.8-28.1$ & $14.0-21.0$ & $40.9-53.6$ & $60.3-69.4$ & $81.5-88.0$ \\
\hline & Yes & $n$ & 278 & 67 & 72 & 178 & 255 & 271 \\
\hline & & $\%$ & 84.4 & 20.7 & 23.7 & 56.8 & 64.9 & 82.3 \\
\hline & & $95 \% \mathrm{Cl}$ & $80.3-88.6$ & $16.6-24.9$ & $18.7-28.6$ & $50.0-63.5$ & $60.3-69.4$ & $78.5-86.1$ \\
\hline & No & $n$ & 7006 & 732 & 468 & 2397 & 6191 & 6986 \\
\hline & & $\%$ & 92.8 & 9.7 & 6.3 & 32.5 & 82.5 & 92.6 \\
\hline & & $95 \% \mathrm{Cl}$ & $92.1-93.5$ & $8.8-10.6$ & $5.5-7.1$ & $29.8-35.2$ & $80.9-84.1$ & $91.8-93.4$ \\
\hline \multirow{9}{*}{$\begin{array}{l}\text { Treated unfairly by a health } \\
\text { professional because of } \\
\text { ethnicity }\end{array}$} & Unsure & $n$ & 922 & 201 & 94 & 309 & 695 & 914 \\
\hline & & $\%$ & 87.6 & 19.3 & 9.6 & 32.8 & 72.2 & 86.7 \\
\hline & & $95 \% \mathrm{Cl}$ & 85.5 - 89.7 & $16.7-21.9$ & $7.8-11.5$ & $29.4-36.2$ & $68.9-75.5$ & $84.3-89.1$ \\
\hline & Yes & $n$ & 273 & 70 & 46 & 120 & 188 & 285 \\
\hline & & $\%$ & 83.1 & 22.2 & 15.9 & 41.3 & 66.4 & 86.9 \\
\hline & & $95 \% \mathrm{Cl}$ & $78.3-87.9$ & $17.7-26.7$ & $11.7-20.1$ & $34.7-48.0$ & $61.1-71.8$ & $82.9-90.9$ \\
\hline & No & $\underline{n}$ & 6876 & 684 & 502 & 2393 & 5828 & 6825 \\
\hline & & $\%$ & 92.9 & 9.3 & 7.1 & 34.4 & 83.0 & 92.4 \\
\hline & & $95 \% \mathrm{Cl}$ & $92.1-93.7$ & $8.4-10.3$ & $6.3-8.0$ & $31.7-37.2$ & $81.4-84.6$ & $91.6-93.2$ \\
\hline \multirow[t]{9}{*}{ Any ethnic discrimination } & Unsure & $n$ & 1020 & 207 & 112 & 359 & 758 & 1000 \\
\hline & & $\%$ & 88.2 & 18.1 & 10.7 & 35.0 & 71.1 & 86.4 \\
\hline & & $95 \% \mathrm{Cl}$ & $86.2-90.3$ & $15.8-20.5$ & $8.9-12.4$ & $31.2-38.8$ & $68.0-74.2$ & $84.1-88.7$ \\
\hline & Yes & $\mathrm{n}$ & 689 & 164 & 111 & 304 & 505 & 696 \\
\hline & & $\%$ & 85.8 & 20.9 & 15.0 & 41.3 & 69.2 & 86.5 \\
\hline & & $95 \% \mathrm{Cl}$ & $83.1-88.4$ & $17.9-23.9$ & $12.0-17.9$ & $36.6-46.0$ & $66.1-72.4$ & $84.2-88.8$ \\
\hline & No & $n$ & 5958 & 542 & 384 & 2094 & 5348 & 5946 \\
\hline & & $\%$ & 93.3 & 8.5 & 6.1 & 33.5 & 84.2 & 93.2 \\
\hline & & $95 \% \mathrm{Cl}$ & $92.6-94.1$ & $7.5-9.4$ & $5.3-6.9$ & $30.7-36.3$ & $82.6-85.8$ & $92.5-94.0$ \\
\hline
\end{tabular}

achievement $(p=0.016)$ variables. Stratification resulted in loss of precision around the odds ratio point estimates in all ethnic groups. Within each ethnic group participants who reported 'yes' or 'unsure' experience of ethnic discrimination were more likely to have experienced significant depressive symptoms and smoke cigarettes at least weekly and less likely to rate their school achievement 'near top/above or about the middle' than those who had not experienced ethnic discrimination. For all three outcomes the direction and size of the association between experience of ethnic discrimination and the outcome were similar across all ethnic groups. (Table 5)

\section{Discussion}

This large, nationally representative study provides prevalence estimates of secondary school student's experience of ethnicity-related bullying at school and their experience of ethnic discrimination by health professionals and by the police. In doing so, it contributes to a 
Table 4 Logistic regressions for associations between experiencing ethnic discrimination and general health, binge drinking and feeling safe in the neighbourhood outcomes and, Youth'07

\begin{tabular}{|c|c|c|c|}
\hline & \multicolumn{3}{|c|}{ Experienced any form of discrimination } \\
\hline & $\begin{array}{c}\text { Unsure } \\
\text { OR } \\
95 \% \mathrm{Cl}\end{array}$ & $\begin{array}{c}\text { Yes } \\
\text { OR } \\
95 \% \mathrm{Cl}\end{array}$ & $\begin{array}{c}\text { No } \\
\text { OR } \\
95 \% \text { Cl }\end{array}$ \\
\hline General health excellent/very good/good & $\begin{array}{c}0.61 \\
0.47-0.78\end{array}$ & $\begin{array}{c}0.51 \\
0.39-0.65\end{array}$ & 1.0 \\
\hline Binge drinking in last 4 weeks & $\begin{array}{c}1.42 \\
1.20-1.68\end{array}$ & $\begin{array}{c}1.77 \\
1.45-2.17\end{array}$ & 1.0 \\
\hline Feels safe in neighbourhood all/most of the time & $\begin{array}{c}0.59 \\
0.51-0.68\end{array}$ & $\begin{array}{c}0.48 \\
0.40-0.58\end{array}$ & 1.0 \\
\hline
\end{tabular}

Adjusted for ethnicity, age, sex, NZDep2006, food security, housing mobility

limited literature about the effect of experiencing ethnic discrimination on the health and wellbeing of young people. It also contributes to a very limited evidence base regarding the effects of ethnic discrimination on the health of indigenous youth.

Māori, Pacific, Asian and Other ethnic group participants were significantly more likely to report experiencing ethnic discrimination than their NZ European peers. Furthermore, the reporting of being unsure about

Table 5 Ethnicity stratified logistic regressions for associations between experiencing ethnic discrimination and depression, smoking and school achievement outcomes, Youth'07

\begin{tabular}{|c|c|c|c|c|c|}
\hline & Māori & Pacific & Asian & Other & NZ European \\
\hline \multicolumn{6}{|c|}{ Significant depressive symptoms in past 12 months } \\
\hline \multicolumn{6}{|l|}{ Yes } \\
\hline OR & 3.35 & 2.94 & 2.28 & 2.77 & 4.10 \\
\hline $95 \% \mathrm{Cl}$ & $1.98,5.67$ & $1.53,5.64$ & $1.55,3.36$ & $1.19,6.45$ & $2.90,5.80$ \\
\hline \multicolumn{6}{|l|}{ Unsure } \\
\hline OR & 1.94 & 1.93 & 2.53 & 1.08 & 2.32 \\
\hline $95 \% \mathrm{Cl}$ & $1.24,3.04$ & $1.07,3.48$ & $1.67,3.83$ & $0.59,1.99$ & $1.69,3.18$ \\
\hline No & 1.0 & 1.0 & 1.0 & 1.0 & 1.0 \\
\hline \multicolumn{6}{|c|}{ Smokes cigarettes at least weekly } \\
\hline \multicolumn{6}{|l|}{ Yes } \\
\hline OR & 3.62 & 1.29 & 1.50 & 3.37 & 4.00 \\
\hline $95 \% \mathrm{Cl}$ & $2.31,5.67$ & $0.62,2.60$ & $0.62,3.64$ & $0.93,12.25$ & $2.62,6.10$ \\
\hline \multicolumn{6}{|l|}{ Unsure } \\
\hline OR & 1.76 & 0.96 & 1.15 & 5.47 & 2.05 \\
\hline $95 \% \mathrm{Cl}$ & $1.16,2,68$ & $0.35,2.60$ & $0.48,2.78$ & $2.23,13.43$ & $1.42,2.96$ \\
\hline No & 1.0 & 1.0 & 1.0 & 1.0 & 1.0 \\
\hline \multicolumn{6}{|c|}{ Achievement near top/above or about the middle } \\
\hline \multicolumn{6}{|l|}{ Yes } \\
\hline OR & 0.44 & 0.77 & 0.93 & 0.37 & 0.52 \\
\hline $95 \% \mathrm{Cl}$ & $0.29,0.67$ & $0.37,1.61$ & $0.47,1.85$ & $0.14,0.97$ & $0.35,0.77$ \\
\hline \multicolumn{6}{|l|}{ Unsure } \\
\hline OR & 0.71 & 0.73 & 0.39 & 0.21 & 0.51 \\
\hline $95 \% \mathrm{Cl}$ & $0.49,1.05$ & $0.42,1.26$ & $0.20,0.77$ & $0.10,0.43$ & $0.35,0.74$ \\
\hline No & 1.0 & 1.0 & 1.0 & 1.0 & 1.0 \\
\hline
\end{tabular}

Adjusted for age, sex, NZDep2006, food security, housing mobility ethnic discrimination was significantly more likely among Māori, Pacific, Asian and Other ethnic groups than the NZ European ethnic group.

Participants who reported 'yes' or 'unsure' to the questions on ethnic discrimination were significantly less likely to report excellent/very good/good health, feel safe in their neighbourhood, and report their achievement at school as about the middle or higher. These participants were also significantly more likely to: have experienced depressive symptoms in the previous 12 months, smoke cigarettes at least weekly, and to have had an episode of binge drinking in the previous 4 weeks. The adverse associations between being 'unsure' about ethnic discrimination and health/wellbeing outcomes were remarkably similar to those who reported 'yes' to experiencing this ethnic discrimination. Our results suggest that for both 'yes' and 'unsure' groups ethnic discrimination may be an important determinant of health and wellbeing.

Youth/school student experience of ethnic discrimination by a health professional has not been, to our knowledge, reported in the literature. However, our findings are consistent with those of the 2002/2003 NZ health survey (participants $\geq 15$ years of age) which found that Māori, Pacific, and Asian adults were significantly more likely to report unfair treatment by a health professional than NZ Europeans [4] and with the international literature that reports that adults from ethnic minorities are more likely to experience differential and unfair treatment by health professionals than dominant ethnic groups [38-41]. Our findings that Pacific, Māori, Asian and Other ethnic group's participants were significantly more likely to report ethnic discrimination by the police is consistent with international literature that shows youth from minority ethnic groups are most likely to report ethnic discrimination or harassment by the police [42-44].

Compared with NZ European students, all other ethnic groups in the current study were more likely to report being bullied at school because of their ethnicity/ culture, with Asians having the highest self-report rates 
of all groups. The Youth2000 survey also found that Asian students were the ethnic group most likely to report ethnicity related bullying in schools, with Pacific then Maori students the next highest groups [45]. A NZ survey of international students (92\% of whom were Asian and most were at secondary school), revealed that less than half believed that New Zealanders have positive attitudes towards international students and a third believed that ethnic discrimination was a common experience among international students [46]. The studies noted above were not designed to explore the reasons for these findings. However, a small pilot study of secondary school students in New Zealand suggests that inter-ethnic 'intimidatory practices' experienced by Asian ethnic minorities may reflect social distance (separation) between Asian and non-Asian ethnic groups and/or the Asian ethnic groups' perceptions about acceptance in the wider community [47]. These issues require further exploration alongside consideration of potential intersecting issues such as the experience of being (in some cases) newer migrants [48]. Literature from the US and the UK also document the prevalence and severity of ethnicity-related bullying among minority ethnic groups in comparison to the dominant ethnic group $[49,50]$.

In NZ, Harris et al. [4] found that adults who reported experiencing ethnic discrimination were significantly more likely to report poor/fair self-rated health, lower physical functioning, poorer mental health, and current smoking. Our findings amongst secondary students were similar, with students who reported ethnic discrimination being more likely to report fair/poor self-rated health, have experienced significant depressive symptoms, and be cigarette smokers. Our findings are also consistent with international reports that, among adult and youth populations, ethnic discrimination is associated with negative self-reported health and poorer overall health status $[9,11-14,51]$, depressive symptoms or depression $[7,13,14,20,52-55]$, smoking [14,20-22,56-58], alcohol use [14,20-22], and use of other substances $[14,20,21,23]$. International literature has also reported that youth who experience ethnic discrimination are more likely to feel unsafe in their neighbourhood because of bullying that started in school and overflowed into the victims' neighbourhood [59] and that those who experience ethnicity-related bullying in schools are more likely to have lower levels of academic achievement [60,61]. While our study used 'any ethnic discrimination' rather than ethnicity-related bullying specifically, the international conclusions are consistent with our findings.

Due to the cross-sectional design of the study we are able to report associations but not attribute causality. Other literature discusses hypothesised mechanisms and pathways between experiencing ethnic discrimination and adverse health outcomes [14,55]. Williams and Mohammed [14] describe three potential pathways through which experiencing ethnic discrimination may adversely affect health. They argue that exposure to stress (ethnic discrimination) results in psychological distress that adversely affects health; that behavioural coping strategies to manage stress may include unhealthy behaviours such as smoking and alcohol misuse; and that psychological and behavioural responses to stressors can lead to structural and functional alterations in physiological systems [14]. Personal and social factors such as the strategies used to cope with stressors, social support, level of vigilance and anticipatory anxiety about ethnic discrimination, ethnic identity, and ethnic group identification may also moderate or mediate the effect of experiencing ethnic discrimination on health outcomes [14,55]. Bals, Turi, Skre and Kvernmo [62] found that 'enculturation factors' such as participation in cultural activities and Sami language competence were associated with decreased mental health problems among Indigenous Sami youth. The interactions between enculturation/cultural resilience, experiencing ethnic discrimination and health outcomes require further elucidation.

This study has a number of strengths: it is a large, nationally representative survey with good school and student response rates. The anonymous survey using $M-$ CASI allowed investigation of sensitive issues that may be under-reported using other data collection methods such as face-to-face interviewing.

There are a number of limitations that should be considered. Firstly, the survey was conducted with young people attending secondary schools. The experiences and health outcomes among young people who are not attending school may systematically differ from those who are attending school [63]. In addition, ethnic discrimination has been described as a significant influence on early school leavers [64]. Māori and Pacific youth in NZ leave school early $[65,66]$ suggesting that the findings in this study may underestimate the prevalence of ethnic discrimination among the 'total' (i.e. including those that are not at school) population of young people in this age group.

Secondly, participants were asked about their experience of ethnicity-related bullying and ethnic discrimination by health professionals and the police. There are a number of other domains where participants may have been exposed to ethnic discrimination, for example by staff at schools and in other social settings, which have not been included in the current study. Furthermore, institutional forms of discrimination are unlikely to have been captured by the measures used in the current study. As a result our findings may underestimate the 
overall experience of ethnic discrimination experienced by participants.

Thirdly, some data may be vulnerable to recall bias. As the accuracy of recall is unlikely to be influenced by the participant's ethnic group, or their experience of ethnic discrimination, it is unlikely that recall bias will substantially alter the effect estimates. Fourthly, the measurement of socio-economic position among adolescents poses challenges as they may be unable to provide accurate information about common measures of socioeconomic position such as parental occupation, education level, or income $[67,68]$. This study used three alternative socio-economic variables; however, there may still be residual confounding by socio-economic position.

Finally, we are unable to ascertain whether students who responded they were 'unsure' were unsure whether they had experienced discrimination, or were unsure whether this was because of their ethnicity. 'Unsure' responses may reflect attributional ambiguity where the experience is ambiguous and difficult for recipients to label as discriminatory [14].

Future research could address ethnic discrimination in the school setting, non-bullying ethnic discrimination by peers, and the nature, frequency and intensity of discriminatory experiences. In addition, studies are needed to clarify the nature of 'unsure' responses and to examine the associations between ethnic discrimination and health/wellbeing outcomes with a view to understanding the pathways between experiencing ethnic discrimination and adverse health and wellbeing outcomes and potential interventions.

\section{Conclusions}

Freedom from ethnic discrimination is embedded with the UN Declaration of Human Rights, the NZ Bill of Rights Act, and other international human rights instruments. In conjunction with the findings relating to the adult population by Harris et al., our findings that secondary school students are cognisant of ethnic discrimination speak to the progress yet to be made in wider society to ensure that these rights are met for all people living in Aotearoa New Zealand.

\footnotetext{
Abbreviations

NZ: New Zealand; NZDep2006: New Zealand Index of Deprivation 2006; RADS: Reynolds Adolescent Depression Scale; RADS-SF: Reynolds Adolescent Depression Scale short form version; US: United States of America; UK: United Kingdom.
}

\section{Acknowledgements}

Members of the Adolescent Health Research Group, Faculty of Medical and Health Sciences. Erica D'Souza assisted with some of the data analysis and interpretation. The Youth'07 national survey of the health and wellbeing of secondary school students in New Zealand was funded by the Health Research Council of New Zealand, the Department of Labour, Families
Commission, Accident Corporation of New Zealand, Sport and Recreation New Zealand, The Alcohol Advisory Council of New Zealand, and the Ministries of Youth Development, Justice and Health.

\section{Author details}

${ }^{1}$ Te Kupenga Hauora Mãori, School of Population Health, Faculty of Medical and Health Sciences, University of Auckland, Private Bag 92019, Wellesley St, Auckland, New Zealand. 'Epidemiology and Biostatistics, School of Population Health, Faculty of Medical and Health Sciences, University of Auckland, Private Bag 92019, Wellesley St, Auckland, New Zealand. ${ }^{3}$ School of Nursing, Faculty of Medical and Health Sciences, University of Auckland, Private Bag 92019, Wellesley St, Auckland, New Zealand.

\section{Authors' contributions}

SC was involved in the conception and design, acquisition of data, data analysis and interpretation, wrote this manuscript, and has given final approval of the version. ER was involved in the conception and design, acquisition of data, data analysis and interpretation, revised the manuscript critically for important intellectual content, and has given final approval of the version. SA was involved in the conception and design, acquisition of data, data analysis and interpretation, revised the manuscript critically for important intellectual content, and has given final approval of the version. TC was involved in the conception and design, acquisition of data, data analysis and interpretation, revised the manuscript critically for important intellectual content, and has given final approval of the version. DR was involved in data interpretation, drafting of the manuscript, revised the manuscript critically for important intellectual content, and has given final approval of the version.

\section{Authors' information}

SC is a Senior Lecturer (Medical) and Director of Tōmaiora Māori Health Research Centre. ER is a Senior Research Fellow. SA is a Professor of Epidemiology. TC is a Senior Lecturer in the School of Nursing. DR is a Research Assistant in the School of Nursing.

\section{Competing interests}

The authors declare that they have no competing interests.

Received: 16 August 2011 Accepted: 18 January 2012

Published: 18 January 2012

\section{References}

1. Bhopal R: Spectre of racism in health and health care: lessons from history and the United States. Br Med J 1998, 316:1970-1973.

2. Williams DR: Race and health: basic questions, emerging directions. Ann Epidemiol 1997, 7:322-333.

3. Greene ML, Way N, Pahl K: Trajectories of perceived adult and peer discrimination among Black, Latino, and Asian American adolescents: patterns and psychological correlates. Dev Psychol 2006, 42:218-236.

4. Harris R, Tobias M, Jeffreys M, Waldegrave K, Karlsen S, Nazroo J: Racism and health: The relationship between experience of racial discrimination and health in New Zealand. Soc Sci Med 2006, 63:1428-1441.

5. Krieger N: Embodying inequality: a review of concepts, measures, and methods for studying health consequences of discrimination. Int $J$ Health Sen 1999, 29:295-352.

6. Karlsen S, Nazroo JY: Relation between racial discrimination, social class, and health among ethnic minority groups. Am J Public Health 2002, 92:624-631.

7. Coker TR, Elliott MN, Kanouse DE, Grunbaum JA, Schwebel DC, Gilliland MJ, Tortolero SR, Peskin MF, Schuster MA: Perceived racial/ethnic discrimination among fifth-grade students and its association with mental health. Am J Public Health 2009, 99:878-884.

8. Virdee S: Racial harassment. In Ethnic minorities in Britain: Diversity and disadvantage. Edited by: Madood T, Berthoud R, Lakey J, Nazroo J, Smith P, Virdee S, Beishon S. London: Policy Studies Institute; 1997:259-289.

9. Nazroo JY: The structuring of ethnic inequalities in health: economic position, racial discrimination, and racism. Am J Public Health 2003, 93:277-284.

10. Larson A, Gillies M, Howard P, Coffin J: It's enough to make you sick: the impact of racism on the health of Aboriginal Australians. Aust N Z J Public Health 2007, 31:322. 
11. Mays VM, Cochran SD, Barnes NW: Race, race-based discrimination, and health outcomes among African Americans. Annu Rev Psychol 2007, 58:201-225.

12. Stuber J, Galea S, Ahern J, Blaney S, Fuller C: The Association between Multiple Domains of Discrimination and Self-assessed Health: A Multilevel Analysis of Latinos and Blacks in Four Low-Income New York City Neighborhoods. Health Serv Res 2003, 38:1735-1760.

13. Priest N, Paradies $Y$, Stewart $P$, Luke J: Racism and health among urban Aboriginal young people. BMC Public Health 2011, 11:568.

14. Williams DR, Mohammed SA: Discrimination and racial disparities in health: evidence and needed research. J Behav Med 2009, 32:20-47.

15. Finch BK, Kolody B, Vega WA: Perceived discrimination and depression among Mexican-origin adults in California. J Health Soc Behav 2000, 41:295-313.

16. Bowen-Reid TL, Harrell JP: Racist experiences and health outcomes: an examination of spirituality as a buffer. J Black Psychol 2002, 28:18-36.

17. Shavers VL, Shavers BS: Racism and health inequity among Americans. J Natl Med Assoc 2006, 98:386-396.

18. Dotterer AM, McHale SM, Crouter AC: Sociocultural factors and school engagement among African American youth: the roles of racial discrimination, racial socialization, and ethnic identity. Appl Dev Sci 2009, 13:61-73.

19. Clark $R$, Anderson NB: Racism as a stressor for African Americans. Am Psychol 1999, 54:805

20. Paradies Y: A systematic review of empirical research on self-reported racism and health. Int J Epidemiol 2006, 35:888-901.

21. Pachter LM, Coll CG: Racism and child health: a review of the literature and future directions. J Dev Behav Pediatr 2009, 30:255-263.

22. Yoo HC, Gee GC, Lowthrop CK, Robertson J: Self-reported racial discrimination and substance use among Asian Americans in Arizona. $J$ Immigr Health Minor Health 2010, 12:683-690.

23. Surko M, Ciro D, Blackwood C, Nembhard M, Peake K: Experience of racism as a correlate of developmental and health outcomes among urban adolescent mental health clients. Soc Work Ment 2005, 3:235-260.

24. Harris R, Tobias M, Jeffreys M, Waldegrave K, Karlsen S, Nazroo J: Effects of self-reported racial discrimination and deprivation on Maori health and inequalities in New Zealand: cross-sectional study. Lancet 2006, 367:2005-2009.

25. Denny SJ, Grant S, Utter J, Robinson EM, Fleming TM, Milfont TL, Crengle S, Clark T, Ameratunga SN, Dixon R, et al: Health and well-being of young people who attend secondary school in Aotearoa, New Zealand: What has changed from 2001 to 2007? J Paediatr Child Health 2011, 47:191-197.

26. Robson B, Purdie G: Mortality. In Hauora: Māori Standards of Health IV A study of the years 2000-2005. Edited by: Robson B, Harris R. Wellington: Te Rōpū Rangahau a Eru Pōmare and University of Otago; 2007:

27. Robson B, Robson C, Harris R, Purdie G: Hospitalisations. In Hauora: Māori Standards of Health IV A study of the years 2000-2005. Edited by: Robson B, Harris R. Wellington: Te Rōpū Rangahau a Eru Pōmare and University of Otago; 2007:

28. Clark TC, Robinson E, Crengle S, Herd R, Grant S, Denny S: Te Ara Whakapiki Taitamariki Youth'07- the Health and Wellbeing of Secondary School Students in New Zealand. Results for Māori young People. Book Te Ara Whakapiki Taitamariki Youth'07- the Health and Wellbeing of Secondary School Students in New Zealand. Results for Mäori young People Auckland: University of Auckland; 2008.

29. Helu S, Robinson E, Grant S, Herd R, Denny S: Youth '07 - the Health and Wellbeing of Secondary School Students in New Zealand Results for Pacific Young People. Book Youth ' 07 - the Health and Wellbeing of Secondary School Students in New Zealand Results for Pacific Young People Auckland: University of Auckland; 2009.

30. Adolescent Health Research Group: Youth'07: The Health and Wellbeing of Secondary School Students in New Zealand. Technical Report. Book Youth'07: The Health and Wellbeing of Secondary School Students in New Zealand. Technical Report Auckland: The University of Auckland; 2008.

31. Denny S, Milfont T, Utter J, Robinson E, Ameratunga S, Merry S, Fleming T, Watson P: Hand-held internet tablets for school-based data collection. BMC Research Notes 2008, 1:52.

32. Statistical Standard for Ethnicity 2005. [http://www2.stats.govt.nz/domino/ external/web/carsweb.nsf/55d63ae38ba3a25e4c2567e6007f6686/ 35d9b7e17a1d6151cc25701100031353?OpenDocument].
33. Ministry of Health: Ethnicity Data Protocols for the Health and Disability Sector. Book Ethnicity Data Protocols for the Health and Disability Sector Wellington: Ministry of Health; 2004.

34. Salmond C, Crampton P, Atkinson J: NZDep2006 Index of Deprivation. Book NZDep2006 Index of Deprivation Wellington: Department of Public Health, University of Otago; 2007.

35. Reynolds WM, Mazza JJ: Reliability and validity of the reynolds adolescent depression scale with young adolescents. J Sch Psychol 1998, 36:295.

36. Walker L, Merry S, Watson PD, Robinson E, Crengle S, Schaaf D: The Reynolds adolescent depression scale in New Zealand adolescents. Aust N Z J Psychiatry 2005, 39:136-140.

37. Milfont TL, Merry S, Robinson E, Denny S, Crengle S, Ameratunga S: Evaluating the short form of the Reynolds adolescent depression scale in New Zealand adolescents. Aust N Z J Psychiatry 2008, 42:950-954.

38. Smedley BD, Stith AY, Nelson AR: Unequal treatment: Confronting racial and ethnic disparities in health care Washington DC: The National Academies Press; 2003.

39. van Ryn M, Burke J: The effect of patient race and socio-economic status on physicians' perceptions of patients. Soc Sci Med 2000, 50:813-828.

40. LaVeist TA, Rolley NC, Diala C: Prevalence and patterns of discrimination among U.S. health care consumers. Int J Health Serv 2003, 33:331-344.

41. Blanchard J, Lurie N: R-E-S-P-E-C-T: patient reports of disrespect in the health care setting and its impact on care. J Fam Pract 2004, 53:721-730.

42. Seaton EK, Caidwell CH, Sellers RM, Jackson JS: The prevalence of perceived discrimination among African American and Caribbean black youth. Dev Psychol 2008, 44:1288-1297.

43. Wortley S, Tanner J: Discrimination or 'good' policing? The racial profiling debate in Canada. Our Diverse Cities 2004, 1:197-201.

44. Rosenbloom SR, Way N: Experiences of discrimination among African American, Asian American, and Latino Adolescents in an urban high school. Youth \& Soc 2004, 35:420-451.

45. Fleming $T$, Watson $P$, Robinson $E$, Ameratunga $S$, Dixon $R$, Clark $T$, Crengle S: Violence and New Zealand young people: findings of youth2000 - a national secondary school youth health and wellbeing survey. Book Violence and New Zealand young people: Findings of Youth2000 - A national secondary school youth health and wellbeing survey Auckland: University of Auckland; 2007.

46. Ministry of Education: The experiences of international students in New Zealand. Book The experiences of international students in New Zealand Wellington: The Ministry of Education; 2004.

47. Sobrun-Maharaj A: The social acceptance of visible ethnic minority adolescents of Asian origin in Auckland secondary schools. Doctor of Philosophy Massey University; 2002.

48. Ameratunga $\mathrm{S}$, Horner J: 'Asian' and immigrant minority youth in Aotearoa/New Zealand. Improving the Transition Reducing the social and psychological morbidity during adolescence A report from the Prime Minister's Chief Scientific Advisor Wellington, New Zealand: Office of the Prime Minister's Science Advisory Committee; 2011.

49. Fisher CB, Wallace SA: Discrimination distress during adolescence. J Youth Adolesc 2000, 29:679.

50. Eslea M, Mukhtar K: Bullying and racism among Asian schoolchildren in Britain. Educational Research 2000, 42:207-217.

51. Williams DR, Neighbors HW, Jackson JS: Racial/ethnic discrimination and health: findings from community studies. Am J Public Health 2003, 93:200-208.

52. Santana V, Almeida-Filho N, Roberts R, Cooper SP: Skin colour, perception of racism and depression among adolescents in Urban Brazil. Child Adolesc Ment Health 2007, 12:125-131.

53. Brody GH, Yi-Fu C, Murry VM, Simons RL, Xiaojia G, Gibbons FX, Gerrard M, Cutrona CE: Perceived discrimination and the adjustment of African American youths: a five-year longitudinal analysis with contextual moderation effects. Child Dev 2006, 77:1170-1189.

54. Szalacha LA, Erkut S, García Coll C, Alarcón O, Fields JP, Ceder I: Discrimination and Puerto Rican children's and adolescents' mental health. Cultur Divers Ethni Minor Psychol 2003, 9:141-155.

55. Pascoe EA, Smart Richman L: Perceived discrimination and health: a meta-analytic review. Psychol Bull 2009, 135:531-554.

56. Bennett GG, Wolin KY, Robinson EL, Fowler S, Edwards CL: Perceived racial/ ethnic harassment and tobacco use among African American young adults. Am J Public Health 2005, 95:238-240. 
57. Landrine $H$, Klonoff EA: Racial discrimination and cigarette smoking among blacks: findings from two studies. Ethn Dis 2000, 10:195-202.

58. Chae DH, Takeuchi DT, Barbeau EM, Bennett GG, Lindsey J, Krieger N: Unfair treatment, racial/ethnic discrimination, ethnic identification, and smoking among Asian Americans in the National Latino and Asian American Study. Am J Public Health 2008, 98:485-492.

59. Nansel TR, Overpeck MD, Haynie DL, Ruan WJ, Scheidt PC: Relationships between bullying and violence among US youth. Arch Pediatr Adolesc Med 2003, 157:348-353.

60. Glover D, Gough G, Johnson M, Cartwright N: Bullying in 25 secondary schools: incidence, impact and intervention. Educ Res 2000, 42:141-156.

61. Spriggs AL, lannotti RJ, Nansel TR, Haynie DL: Adolescent bullying involvement and perceived family, peer and school relations: commonalities and differences across race/ethnicity. J Adolesc Health 2007, 41:283-293.

62. Bals M, Turi AL, Skre I, Kvernmo S: The relationship between internalizing and externalizing symptoms and cultural resilience factors in Indigenous Sami youth from Arctic Norway. Int J Circumpolar Health 2011, 70:37-45.

63. Guttmacher S, Weitzman BC, Kapadia F, Weinberg SL: Classroom-based surveys of adolescent risk-taking behaviors: reducing the bias of absenteeism. Am J Public Health 2002, 92:235-237.

64. Stone S, Han M: Perceived school environments, perceived discrimination, and school performance among children of Mexican immigrants. Child Youth Serv Rev 2005, 27:51-66.

65. Stand downs, suspensions, exclusions and expulsions from school. [http://www.educationcounts.govt.nz/indicators/main/student-engagementparticipation/80346].

66. Robson B, Cormack D, Cram F: Social and economic indicators. In Hauora: Mäori standards of health IV A study of the years 2000-2005. Edited by: Robson B, Harris R. Wellington: Te Rōpū Rangahau Hauora a Eru Pōmare; 2007:

67. Wardle J, Robb K, Johnson F: Assessing socioeconomic status in adolescents: the validity of a home affluence scale. J Epidemiol Community Health 2002, 56:595-599.

68. Currie CE, Elton RA, Todd J, Platt S: Indicators of socioeconomic status for adolescents: the WHO health behaviour in school-aged children survey. Health Educ Res 1997, 12:385-397.

\section{Pre-publication history}

The pre-publication history for this paper can be accessed here:

http://www.biomedcentral.com/1471-2458/12/45/prepub

doi:10.1186/1471-2458-12-45

Cite this article as: Crengle et al.: Ethnic discrimination prevalence and associations with health outcomes: data from a nationally representative cross-sectional survey of secondary school students in New Zealand. BMC Public Health 2012 12:45.

\section{Submit your next manuscript to BioMed Central and take full advantage of:}

- Convenient online submission

- Thorough peer review

- No space constraints or color figure charges

- Immediate publication on acceptance

- Inclusion in PubMed, CAS, Scopus and Google Scholar

- Research which is freely available for redistribution

Submit your manuscript at www.biomedcentral.com/submit
Biomed Central 